On Filipino Philosophy

\title{
Ferriols at Heidegger: Ang Pagbigkas sa Kasaysayan bilang Talagang Nangyayari
}

Marc Oliver D. Pasco

\begin{abstract}
This essay aims to view the philosophy of Roque J. Ferriols, from a Heideggerian perspective. It argues that Ferriols' way of philosophizing may be appreciated by illuminating it with Heidegger's authentically historical way of philosophizing. Heidegger's Wiederholung or creative retrieval of Ancient Greek philosophy is evident in Ferriols' own approach in thinking meron. Both hold that genuinely historical philosophical thinking may not be reduced to a historiographical approach to philosophizing, akin to Nietzsche's account of the antiquarian approach to history. To wit, it may be argued that Ferriols' way of thinking is somehow similar to Heidegger's insofar as it desires to perform its own unique way of creatively retrieving the meaning of meron from its authentically historical roots as talagang nangyayari, as thought and lived out in the Filipino context. Ferriols' pagbigkas sa meron resonates with Heidegger's call to retrieve the historically forgotten meaning of Sein.
\end{abstract}

Keywords: kasaysayan, talagang nangyayari, Wiederholung, pagbigkas sa meron

\section{Si Ferriols bilang Istoryador ng Pilosopiya}

$\mathrm{M}$ alimit na nauunawaan ang kasaysayan bilang proseso ng paglipas ng panahon mula sa nakaraan, ang paghantong nito sa ngayon, at ang pagdako nito sa hinaharap. Madalas ding mas nabibigyangdiin ang yugto ng nakaraan sa prosesong ito, dulot na rin siguro ng pagkilala na sadyang mas may halaga ang mga bagay at pangyayaring hindi na maibabalik o mababalikan pa kaysa sa mga pangyayaring hawak pa at mababago pa sa ngayon at lalo na sa mga pangyayaring wala pa naman, at kung gayo'y hindi pa dapat isipin. Kung iisipin, ang ganitong pasulong at romantikong interpretasyon sa kasaysayan ay batay sa perspektibo ng tao bilang tagapagmasid ng mga pangyayari mula sa isang abot-tanaw na animo'y nasa labas o sumasaibayo sa kasaysayan mismo. Nahihiwalay ang

(C) 2018 Marc Oliver D. Pasco

https://www.kritike.org/journal/issue 23/pasco december2018.pdf

ISSN 1908-7330

(cc) $\mathrm{BY}-\mathrm{NC}-\mathrm{ND}$ 
tao sa kasaysayan sa ganitong pagtanaw. Hindi niya ito itinuturing bilang siyang mismong kinikilusang abot-tanaw ng lahat ng tungkol sa kanya, bagkus ay tinititigan niya ito sa mga pahina ng makakapal na libro ng kasaysayan, o 'di kaya'y sa mga webpage sa internet, na siyang nagtataguyod ng akademikong pagtingin sa kasaysayan bilang isang kuwentong binabasa ng isang mambabasa. Kung mananatili lamang tayo sa ganitong pananaw, hindi natin lubusang mapapahalagahan ang ambag ni Roque Ferriols sa kasaysayan, hindi lamang ng pilosopiyang Filipino, kundi sa pilosopiya mismo.

Sa bungad ng kanyang akdang Mga Sinaunang Griyego, inilista ni Ferriols ang mga kakailanganing katangian at kakayahan ng isang istoryador ng pilosopiyang handang makipagsapalaran at umunawa sa mga katutubong kaisipan ng mga sinaunang palaisip. ‘Ika niya:

Ang nasabing istoryador ay nangangailangan ng: 1) pagkaeksakto: nang huwag niyang baluktutin o pilipitin ang talagang iniisip ng pilosopong kanyang pinagaaralan 2) buhay na pagmumulat na kapuwa tao rin ang mga pilosopo 3) pagsisikap maging maunawain sa mga taong marahil ibang-iba sa kanya ang uri ng pag-iisip 4) pananabik matulungang magbukas ng kalooban nang magamit niya, ika nga, ang mga bahagi ng kanyang utak at puso na hindi niya nakaugaliang gamitin. ${ }^{1}$

Sa siping ito, malinaw ang iginagawad na pagpapahalaga ni Ferriols sa pagiging mulat at bukas sa abot-tanaw ng mga pilosopong ninanais nating maunawaan. Bukod sa kahilingang maging eksakto o tapat sa binigkas ng mga pilosopo ayon sa pag-unawa natin sa kanila bilang kapwa-taong may sariling ginagalawang konteksto, kapansin-pansin ang pagpapahalaga ni Ferriols sa papel ng istoryador bilang bukas, buhay, at dinamikong mambabasang handang ilagay ang kanyang sarili sa alanganin at paganahain ang mga bahagi ng kanyang pag-iisip at pag-unawang hindi niya kinasanayang gamitin alang-alang sa pagiging tapat sa pakikipagsapalaran sa kasaysayan. Wari ba'y pinaaalahanan tayo ni Ferriols na ang pakikipagsapalaran sa kasaysayan ng pilosopiya ay isang galaw ng sariling ginagabayan hindi lamang ng personal na kahiligan kundi ng mismong mga bukal ng pag-uunawang may kakayahan pa ring makipag-usap sa atin, sa ngayon maging, sa hinaharap. Para sa kanya, ang mga labi nina Herakleitos, Parmenides, Xenophanes, Platon, at Aristoteles ay mga buhay na

${ }^{1}$ Roque J. Ferriols, Mga Sinaunang Griyego (Quezon City: Office of Research and Publications, Ateneo de Manila University, 1991), 2. 
pagpapahayag ng mga diwang buhay na buhay pa rin sa ngayon. Hindi niya ito inaral dahil itinuring niya ang mga itong antigong labi na pinaka-iingatan niyang mapanatili sa kanilang dalisay na estado bilang luma at gayo'y mahalaga. Ang halaga ng mga labi ay nababatay sa kakayahan ng mga itong pukawin ang isip at diwa ng istoryador nang mabigkas niya ang meron nang ayon sa wasto nitong abot-tanaw.

Ang problematikong nais tugunan ng papel na ito ay ang paksang may kinalaman sa reduksyon ng kasaysayan sa ideya ng mala-Almanac na tambakan ng kronolohikal na pangyayari, hindi naiiba sa karakterisasyon ni Nietzsche sa kanyang maikling librong, On the Uses and Disadvantages of History for Life, sa antikwaryong pagtanaw sa kasaysayan. Ang ganitong pananaw ay nagpapa-bangkay sa kasaysayan, itituturing ito bilang isang ispesimen na binabalangkasan at pinapatawan ng mga makataong panukat na hindi kumikilala sa likas nitong wika at dinamismo. Sa palagay ko, makatutulong ang gagawing sabayang pagtalakay sa mga pilosopiya nina Ferriols at Heidegger sa pagbuhay sa intrinsikong dinamismo ng kasaysayan kapag ito'y inunawa sa lilim ng kanilang pag-unawa sa meron at Sein at ang relasyon nito sa makasaysayang pag-iral ng tao.

Nais ipakita ng sanaysay na ito na ang pamimilosopiya ni Ferriols ay tunay na makasaysayan sa ganang ito'y buhay at dinamikong pakikipagtalastasan hindi lamang sa mga sinaunang pilosopo, kundi sa meron mismo na patuloy na kumikilos at sumasanib sa ginagawa niyang pamimilosopiya. Sa gagawing pagtanaw sa pilosopiya ni Ferriols mula sa lente ng pamimilosopiya ni Martin Heidegger, susubuking ipakita ang pagkakahawig ng kanilang pamamaraan ng pamimilosopiya mula sa perspektibo ng pag-unawa sa kasaysayan bilang pakikipagsapalaran sa pamamagitan ng malikhaing pag-uulit sa pangyayari ng talagang nangyayari. Susubukin ding tingnan kung mapapalalim ni Ferriols ang diskurso ni Heidegger tungkol sa panahon ng modernong teknolohiya. Sa huli, layunin ng papel na ito na itaguyod ang isang larawan ni Ferriols bilang isang palaisip na tunay na kumilos sa abot-tanaw ng meron dahil siya ay nakipagsapalaran sa isang makasaysayang paraan sa talagang nangyayari at hindi lamang nagpa-akit sa kasiguruhan at malilinaw na hangganan ng konsepto. Gaya ng mismong sabi niya:

Ang pag-aalala naman sa meron ay pagbabalik sa isang pagmamalay-tao, na hindi iniwanan kailan man. Ano itong pagmamalay-tao na hindi iniwanan? Ang pagbigkas sa meron. Hindi ko ibig sabihin na kailangang sabihin ninoman: meron, merong ganito, merong ganoon. Ang buhay ninoman na mulat at gising sa talagang nagyayari ay pagbigkas sa meron. At mulat

(C) 2018 Marc Oliver D. Pasco

https://www.kritike.org/journal/issue 23/pasco december2018.pdf ISSN 1908-7330

(c) $)$ BY-NC-ND 
siya sa isang malalim, hindi-konsepto at lihim na pagmamalay na binibigkas nga niya ang meron. ${ }^{2}$

Para kay Ferriols ang pag-aalala ay isang kilos ng pagbabalik sa mismong sarili ng tao. Kapag inaalala natin ang meron sa pamamagitan ng pagbigkas dito, nangyayari ang pagiging tao ng ating pagkatao at nakikisalamuha't sumasabak tayo sa daluyong ng kasaysayang patuloy na humahamon sa ating lumikha ng isang matinong sariling marunong bumigkas sa wika ng meron na talagang makasaysayan.

\section{Ang Meron bilang Paksang Historikal: Pag-uulit ayon kina Ferriols at Heidegger}

Para sa palaisip na si Martin Heidegger, mainam na linawin ang pagkakaiba ng kahulugan ng dalawang salitang Aleman para sa kasaysayan, ang Historie at Geschichte. Ang Historie ay tumutukoy sa kasaysayan bilang historiyograpiya o ang disiplinado at siyentipikong pag-aaral sa mga pangyayari sa nakaraan at ang mga kaugnayan ng mga ito, samantalang ang Geschichte ay may kinalaman sa mismong pangyayari ng mga pangyayari batay sa "bigay" o pagtatakda ng Sein o ng pagprepresensya ng nagprepresensya. Ayon kay Heidegger, "chronological distance and causal sequence do indeed belong to the study of historiography, but are not themselves history." ${ }^{3}$ Ang kasaysayan, bilang Geschichte ay ang kasaysayan ng Sein o Seinsgeschichte. Inungkat ni Heidegger ang etimolohikal na relasyon ng salitang Geschichte sa Geschick na nangangahulugang "destining" o pagtatakda. Ayon kay William Lovitt, isang tagapagsalin ng mga akda ni Heidegger, "the word Geschick can mean fate or destiny. Furthermore, the word Schickung, which denotes a 'providential decree or dispensation,' is closely related to Geschick. Another term, the verb, sich schicken, which means 'to come to pass' or 'happen,' 'to suit or to be fit' is also related to Geschick." ${ }^{4}$ Mula sa pananaw na ito, masasabing para kay Heidegger, ang kasaysayan, bilang kasaysayan ng

\footnotetext{
2 Roque J. Ferriols, SJ, Pambungad sa Metapisika (Quezon City: Office of Research and Publications, Ateneo de Manila University, 1997), 13.

3Martin Heidegger, "The Anaximander Fragment," in Early Greek Thinking, trans. by David Farrell Krell and Frank Capuzzi (San Francisco: Harper San Francisco, 1984.), 26. Dagdag pa ni Michael Inwood, “The German language has two words for 'history', Geschichte and Historie. Both refer ambiguously to history as events or happenings and to history as the study of events. Heidegger disambiguates them, assigning Geschichte to events, 'history', and Historie to the study of events, 'historiology, the 'historian.' His choice is not arbitrary: Geschichte comes from geschehen, 'to happen', while Historie comes from the Greek historein, 'to enquire, etc."' Michael Inwood, A Heidegger Dictionary (Malden: Blackwell Publishers, 1999), 90.

${ }^{4}$ Martin Heidegger, "The Turning," in The Question Concerning Technology and Other Essays, trans. by William Lovitt (New York: Harper and Row Publishers, 1977), 37.
} 


\section{FERRIOLS AT HEIDEGGER}

Sein ay may kinalaman sa pangyayari ng mismong Sein bilang paglalantadsabay-kubli ng pagprepresensya ng nagprepresensya na siyang kondisyon ng posibilidad ng anumang ispesikong pangyayari sa kasaysayan mula sa lente ng historiyograpiya.

Ang Sein ay nangyayari, bagamat nakakubli. Sa pagkakakubli ng paprepresensya sa taong nakatanod dito, nahuhulog ang tao sa kapalaran ng paglimot (Seinsvergessenheit) dahil natututok ang kanyang atensyon sa mga nagprepresensya at hindi sa mismong pagprepresensya ng mga ito. Kumbaga, batay sa pag-unawa sa kasaysayan bilang Geschichte, malinaw ang kakayahan ng taong umunawa at makisangkot sa mga pangyayari sa kasaysayan gaya ng mga rebolusyon at digmaan, ngunit salat siya sa kamulatan sa saysay ng mga ito sa abot-tanaw ng mismong pangyayari ng mga ito na nababatay sa laro ng litaw-sabay-kubli ng katotohanan o alêtheia. Hindi lubusang kakulangan o kasalanan ng tao ang kanyang paglimot, kundi ito ay buhat sa mismong esensya ng Sein bilang siyang nagkukubli ayon sa pagtatakda na rin Geschick ng Sein mismo. Paglilinaw ni Heidegger:

When we use the word Geschick in connection with be-ing, then we mean that being hails us and clears and lights itself, and in clearing it furnishes the tem-poral play-space wherein beings can appear. In light of the Geschick of being, the history of being is not thought of in terms of a happening characterized by a passing away and a process. Rather, the essence of history is determined on the basis of the Geschick of being, of being as Geschick, of what as such proffers itself to us in withdrawing. Both-proffering and withdrawingare one and the same, not two different things. ${ }^{5}$

Ang nauunang pagtatakda ng Sein ang naghahanda ng espasyo at panahon para sa pangyayari ng kasaysayan. Sa pangyayari ng kasaysayan bilang Geschichte nagiging posible ang pakikipagtagpo sa mga umiiral na siya namang kinakanlong ng kanyang pag-uunawa. Para kay Heidegger hindi mauunawaan ang esensyal na kahulugan ng Sein (na siyang proyekto ng kanyang pamimilosopiya) nang hindi sabay inuunawa ang esensyal na kahulugan ng kasaysayan bilang ang pangyayari ng katotohanan. Wika niya, "History means, again at first appearance arbitrarily, the happening [Ereignis] of a decision about the essence of truth. The manner in which the whole of beings is

${ }^{5}$ Martin Heidegger, The Principle of Reason, trans. by Reginald Lilly (Indianapolis: Indiana University Press, 1996), 62.

(c) 2018 Marc Oliver D. Pasco

https://www.kritike.org/journal/issue 23/pasco december2018.pdf

ISSN 1908-7330

(c) $)$ BY-NC-ND 
revealed, in which man is allowed to stand in the midst of this revelation, is grounded and transformed in such a decision." 6

Sa madaling salita, ipinaliliwanag ni Heidegger na hindi tulad ng kinasanayang kaisipan, hindi tao ang ultimong tagapagtakda ng saysay ng kasaysayan, bagkus, ang anumang interpretasyon at pag-uunawa niya sa mga pangyayaring kinasasangkutan niya sa kasaysayan ay masasabing tugon sa nauna nang pagtatakda ng Geschick ng Sein na nagtataguyod ng parametrong bumabalangkas sa mga bagay na kanyang pag-uukulan ng pansin at isasaloob pati na sa mga aspeto ng mga bagay at pangyayaring hindi niya mapapansin. Bilang halimbawa, sa panahon ng modernong teknolohiya, ang saysay ng mga bagay ay batay sa kanilang pagiging handang magamit at pagkakitaan. Walang kakayahan ang tao na bumaling sa ibang aspeto ng mga nagprepresensya (gaya ng angking 'di matatawarang halaga at ganda ng kalikasan) dahil nakakahon ang kanyang kakayahang pumansin at umunawa sa kuwadro ng mismong yugto ng kasaysayan kung saan ang mga umiiral ay tinitingnan lamang bilang likas-yaman o puhunan.

Kung gayon, masasabi na para kay Heidegger, ang kasaysayan ng Sein ay ang kasaysayan ng paglimot ng tao sa mismong bukal ng kasaysayan na walang iba kundi ang Sein mismo. Sa bawat yugto ng kasaysayan, mula sa panahon ng mga sinaunang Griyego, sa panahong Medyibal, sa panahon ng Aufklärung, hanggang sa panahon ng modernong teknolohiya at ngayon sa panahon ng impormasyon, ipinagkakait ng Sein ang sarili nito (epoché) sa kilos ng pagkukubli pabor sa paglalantad ng mga umiiral nang ayon sa espiritu ng panahong itinakda ng Geschick ng Sein mismo. Nasa mismong esensya (das Wesen) ng Sein ang pagkanakakubli nito mula sa pag-iisip ng tao, at mula sa esensyang ito nakaugat ang panawagan sa malikhaing pag-uulit at pag-aalala (Wiederholung) sa Sein bilang yaong palaging nagkukubli.

Wiederholung o malikhaing pag-uulit at pag-aalala ang paraan ng pag-iisip ni Heidegger sa kasaysayan ng Sein. Ito ay ang kumakatawan sa kanyang pagsusumubok makipagsapalaran sa kasaysayan ng Sein bilang pangyayaring itinatakda ng pagkukubli ng mismong dapat pag-isipan. Ang Wiederholung o pagsundo (holen) muli (wieder) ay ang muling pagtatanong sa pundamental na tanong na parating nalilimutang itanong sa awtentikong paraan sa bawat yugto ng kasaysayan: Ano ang Sein? ${ }^{7}$ Nais niyang muling

${ }^{6}$ Martin Heidegger, Basic Concepts, trans. by Gary E. Aylesworth. (Indianapolis: Indiana University Press, 1993), 17.

7 Isinasalin ko ang Wiederholung na may literal na kahulugang "to fetch again," bilang pagsundong muli sapagkat mababakas sa pilosopiya ni Heidegger lalo na sa mga sanaysay niya sa librong Introduction to Metaphysics at Early Greek Thinking na ang kanyang pakikipagtapatang muli sa mga sinaunang Griyego gaya nina Anaximander, Heraclitus, at Parmenides ay naglalayong ipakita na marami pang posibilidad ang nagkukubli't dapat hayaang marinig muli ayon sa sariling huni ng katutubong wika na maaaring gamitan ng awtentikong pageetimolohiya ng mga salita gaya ng logos at einai. Sinusundong muli ni Heidegger ang naririto 


\section{FERRIOLS AT HEIDEGGER}

sunduin ang tanong na ito mula sa historikal na bukal ng tanong sa panahon ng mga sinaunang Griyego tulad nina Herakleitos at Parmenides hindi dahil sa isang romantikong pagbibigay-halaga sa simula dahil sa pagiging simula nito kung hindi dahil nais niyang pakinggan ang orihinal na abot-tanaw ng tanong mula sa mga labi ng mga unang palaisip na hindi lamang nag-isip kundi dumanas nito upang makapagsimula tayong, na nasa ngayon, na makipagsapalaran at makisangkot sa pundamental na tanong nang may pagkilala at pag-amin sa ating sariling pagkalimot. Sabi niya, "But we do not repeat a beginning by reducing it to something past and not known, which need merely be imitated; no, the beginning must be begun again, more radically, with all the strangeness, darkness, insecurity that attend a true beginning." ${ }^{8}$ Sa palagay $\mathrm{ni}$ Heidegger, sa pamamaraang ito lamang mabubuksan ang ibang posibilidad at paraan ng pakikisangkot natin sa kasaysayan ng mga umiiral at ng Sein mismo dahil sa paraang ito lamang tayo magiging bukas sa mga hindi pa nabigkas at hindi pa nasabi sa kasaysayan ng Sein dahil na rin sa esensya nito bilang nagkukubli. Paliwanag ni George Seidel:

Thus does Heidegger return to the thought of Parmenides and Heraclitus; not simply because their thought still supports our world, but also because in understanding them we understand where thinking with reference to being-for this is how one comes to stand authentically in his philosophical traditionsomehow went off-track; we must also return to the PreSocratics, says Heidegger, if we are to build historically anew. This return to tradition is no mere restoration or uncreative imitation. Heidegger is not striving to promote some sort of Renaissance of pre-Socratic thinking, even if this would be possible. He studies Parmenides and Heraclitus in an attempt to regain our authentic origins, such that our own thinking may be projected in an authentically historical manner into the future. ${ }^{9}$

\footnotetext{
na sa ngayon mula sa nakaraan upang bigyan ng panibagong pagdanas ang tao sa mga simpleng salitang ito na matagal nang natabunan ng tradisyunal na metapisikal na pag-iisip mula pa kay Platon hanggang kay Nietzsche. Kapag sinabing sinusundo muli ang isang tao, halimabawa, may implikasyon na parang may naiwan o nakaligtaan pa o 'di kaya'y naihatid siya sa maling lugar at kailangang dalhin sa orihinal na destinasyon. Sa parehong paraan, sinusundo muli ni Heidegger ang mga binigkas na kataga ng mga sinaunang Griyego upang makahanap ng mas angkop na landas para sa mga ito sa konteksto ng ngayon.

${ }^{8}$ Martin Heidegger, An Introduction to Metaphysics, trans. by Ralph Manheim (New York: Yale University Press, 1959), 39.

${ }_{9}^{9}$ George Joseph Seidel, Martin Heidegger and the Pre-Socratics (Lincoln: University of Nebraska Press, 1964), 121.
}

(c) 2018 Marc Oliver D. Pasco

https://www.kritike.org/journal/issue 23/pasco december2018.pdf

ISSN 1908-7330

(c) BY-NC-ND 
Ganito ang pamamaraan ng makasaysayang pakikipagsapalarang ginawa ni Heidegger sa kanyang pamimilosopiya. May malinaw siyang pagkilala sa mga nakatagong posibilidad na ikinukubli ng kasaysayan sa pag-iisip ng pilosopo at nakita niyang obligasyon ng mga nag-iisip na hayaang makahinga at makapagsalita ang mga nalimutang kahulugan ng mga binigkas ng mga sinaunang palaisip. Para sa kanya, ang mga simpleng kataga, gaya ng esti gar einai ni Parmenides at logos ni Herakleitos ay mga makasaysayang pagbigkas ng tao sa Sein. Sa madaling salita, nais ni Heidegger na tumalab muli ang esensyal na kahulugan ng Sein sa atin dito sa ngayon sa pamamagitan ng malikhaing pag-uulit ng mga tanong na binigkas sa nakaraan nang sa gayo'y maging tunay na makasaysayan ang ating pakikipagsapalaran patungo sa ating hinaharap.

Kung babasahin ang Mga Sinaunang Griyego at Pambungad sa Metapisika ni Ferriols sa lilim ng pagtanaw ni Heidegger sa makasaysayang pagbigkas sa Sein, mababakasan natin ang hawig na pamamaraan ng pakikisangkot sa kasaysayan ng pamimilosopiya. "Ika niya, "Ugali ng mga namimilosopiya na bumuo ng bagong salita, dala ng pangangailangan ng udyok ng pag-iisip. Ngunit, madalas, hindi sila nag-iimbento ng bagong huni, kundi kumukuha ng dati at kilalang huni at ginagamit ito sa bagong paraan. At ang bagong paraan ay may kaugnayan sa mga nakaugalian nang paraan, ngunit iba pa rin. At iba sapagkat iyan ang hinihingi ng paguunawa." ${ }^{10}$ Dagdag pa niya:

Ang sining ng pamumuhay na mulat sa meron, ay sining ng wasto at buhay na pagtatanong. Sa panahon na isinisilang ang isang wika, ang mga taong lumilikha't humuhubog sa wika ay nakatatanggap ng biyaya ng bago at sariwang pagkalito sa meron, ng orihinal na pagtatanong. Ang biyayang ito'y nag-iiwan ng bakas sa mismong wika. Ang buod ng bawat wika ay ilan mga kataga na mga labi, mga bakas, ng sinaunang pagtatanong. At sinumang gumagamit ng anomang wika, ay may kakayahang magtiyagang hanapin ang mga katagang buod, upang buhayin muli ang sinaunang paghahanap at pagkalito na nakatago sa mga katagang iyon ... parang apoy na nakatago sa baga. At maaaring ulitin ang mga mga katagang iyon.

Ganito ang pag-uulit sa wika: hanapin, gisingin, pairalin ang pagtataka, ang mapaglikhang pagkalito na nakatago

${ }^{10}$ Roque J. Ferriols SJ, Pambungad sa Metapisika, 113.

(c) 2018 Marc Oliver D. Pasco https://www.kritike.org/journal/issue 23/pasco december2018.pdf ISSN 1908-7330 


\section{FERRIOLS AT HEIDEGGER}

sa mga katagang buod. Magiging bago at sariwa uli ang wika, Matutuklasan ng umuulit na ang wika ay potensyal sa pag-unawa at paglikha sa meron. Magiging bago at sariwa ang pakikihalubilo sa meron ng taong umuulit sa anomang wika." 11

Inaalingawngaw ng pahayag nito ang sinabi ni Heidegger na, "ways of thinking hold within them that mysterious quality that we can walk them forward and backward, and that indeed only the way back will lead us forward." 12

Sa pakikipagbuno ni Ferriols sa mga labi ng mga palaisip tulad nina Parmenides at Herakleitos, sinubukan niyang tapat na bigkasin muli ang mga nabigkas na sa ngalan ng pagtalima niya sa meron na siyang paksa ng mga binigkas ng mga palaisip ng sinaunang panahon. Maingat niyang hinimay ang bawat taludtod ng mga tula ni Parmenides at sinikap humabi ng isang makasaysayang kuwento mula sa mga ito na tigib ng mga tinawag niyang pahiwatig o hudyat. Nabakasan niya ang pagsusumubok ni Parmenides na gumamit ng talinghaga sa pagbigkas sa meron na hindi kayang sabihin sapagkat sadyang tigib-apaw ang meron. ${ }^{13}$ Paano nasabing makasaysayan ang muling pagbigkas ni Ferriols sa mga nasabi na ng sinaunang pantas? Sapagkat sa kanyang pagsusumubok makipagsapalaran sa isang banyagang abot-tanaw at kausapin ito mula sa abot-tanaw ng Filipino, masasabing lumikha si Ferriols ng sariwang paraan ng pagtataka at pag-uusisa sa isang paksang kung tutuosin ay luma at lipas na. Sa kanyang pagsundo muli sa mga kataga ng sinaunang Griyego, sumibol ang isang sariwa at malikhaing pakikipagtapatan ng diwang Filipino sa diwa ng mga sinaunang Griyego. Sa kanyang pagbabalangkas ng mga labi ni Herakleitos na kilala sa pagiging malabo (ho skoteinos), nabuhay muli ang diwa ng logos para sa atin sa kasalukuyang panahon at natanto natin na may nabubuo nga sa paghihidwaan. Ang metodo ng pag-uulit ni Ferriols ay may kinalaman sa pag-angkas sa mismong abot-tanaw ni Herakleitos at sa huli'y subuking gumalaw sa abot-tanaw ng mismong meron. ${ }^{14}$ Ang logos na siyang sinaunang pagtitipon (legein) na nagbibigay saysay sa pagpapakita ng mga naghihidwaan ay ang mismong gabay ni Ferriols sa kanyang pagsasalin at pakikipag-diskurso sa mga labi. Sa madaling salita, kumilos ang logos sa bigkas ni Ferriols kaya't masasabing tunay na pangyayari ang naganap sa kanyang pag-iisip - talagang nangyari at nangyayari pa rin ang mga bigkas ni Ferriols--makasaysayang tunay. Naging bago at sariwa muli ang wikang

\footnotetext{
${ }^{11}$ Ibid., 43. Ang italisasyon at ellipses ay kay Ferriols.

${ }^{12}$ Martin Heidegger, "A Dialogue on Language," in On the Way to Language, trans. by Peter D. Hertz (New York: Harper and Row Publishers, 1971), 12.

${ }^{13}$ Ferriols, Mga Sinaunang Griyego, 50-68.

${ }^{14}$ Ibid., 27.
}

(C) 2018 Marc Oliver D. Pasco

https://www.kritike.org/journal/issue 23/pasco december2018.pdf

ISSN 1908-7330

(c) BY-NC-ND 
kanyang kinaharap (wikang Griyego) at kasabay nito, nanariwa rin ang wikang Filipino na sumubok umangkas sa abot-tanaw ng wika ng logos ng Sein noong unang panahon. At dito napapatunayan ang sinabi ni Ferriols na, "Walang labas ang abot tanaw ng meron; o, kung gusto mo, walang tunay na labas ang meron. Ang maaari lamang mangyari ay baka ako matauhan na nasa meron na nga pala ako. At magtataka ako na lalo pala itong mahiwaga kaysa sa inakala ko." 15

Binibigkis sina Herakleitos at Parmenides, ng mismong meron sa mga pahiwatig nito na mababakasan sa mismong mga pagtatangkang pagbigkas ng tatlong palaisip. Meron ang paksa ng logos ni Herakleitos, meron din ang tinutukoy ng perpektong bilog ni Parmenides, kung kaya't si Ferriols, na imbestigador ng meron, ay madaling naka-angkas sa ibig nilang sabihin. Lahat sila ay nakipagsapalaran sa kasaysayan, sa esensyal nitong kahulugan bilang pangyayari. Ang pangyayari ng meron ay walang iba kundi ang paglalantad-sabay-kubli ng meron mismo na humihiling ng makasaysayang pagbigkas sapagkat tanging meron lamang ang paksa ng kasaysayan, ang paksa ng mga paksa. Sabi ni Ferriols, "Kapag nasakyan na natin ang meron bilang paksa, makikita natin na ang meron ang dapat tawaging tunay na paksa; paksa ng mga paksa; mas sinauna kaysa sa anomang sinauna. Sapagkat lahat ng mga ibang paksa: maganda, mabuti, totoo, tao, hayup, musika, salita at kahit na ano ... ay paksa lamang kung meron at palibhasa'y meron. Kung hindi meron, wala. At ang wala ay wala." ${ }^{16}$ Sa makasaysayang pagbigkas sa meron, nailalantad ng wika ang nakakubling misteryo ng meron na siyang paksa ng lahat ng paksa. Nahahayaang tipunin ng logos ang mga paghihidwaang bumubuo at lumilinaw ang papel ng tao sa pangyayaring ito na siyang bumubuo sa kasaysayan sa esensyal nitong kahulugan bilang saksi at daluyan ng wika ng meron na maipapamalas niya hindi lamang sa salita kundi mas lalong higit sa paggawa. Tulad ng paalala ni Ferriols, "Ang pagbigkas na tugon sa tawag ng meron, hindi maaaring manatili sa isip lamang. Kailangang gumagawa. Kung walang paggawa, walang pagkataya." 17 Sa palagay nina Ferriols at Heidegger, ang pagkatao ng tao ay nakaugat sa kanyang kakayahang tumayong saksi sa meron at mabuhay nang nababatay sa pahiwatig ng meron sa pamamagitan ng malikhaing pag-uulit ng mga katutubong pagbigkas sa meron na hindi maikukulong kailanman sa anumang batayang konspeto. Wika ni Ferriols:

Meron nang pag-iisip na nangyari sa nakaraang panahon, pero ang pag-iisip na nangyari na ay maaaring

\footnotetext{
${ }^{15}$ Ferriols, Pambungad sa Metapisika, 11.

16 Ibid., 128. Ang ellipses ay kay Ferriols.

17Tbid., 119.
} 
pangyarihin muli. Ang mga nangyari sa larangan ng diwa ay hindi natatapos kailanman. At sa taong buhay, hindi naluluma kailanman ang anumang ideya. At kaya nga kung ang isang tao ay makikihalubilo sa isang buhay na pag-iisip, kahit na iyong nag-isip ay namatay na, pero ang kanyang pag-iisip ay buhay pa, kung makikihalubilo ka roon at pangyarihin mo muli, mabubuhay iyon uli sa iyong pag-iisip, at marami kang mauunawaan, marami kang makikita na hindi mo sana makikita kung hindi dahil sa kanya. ${ }^{18}$

Maaaring pangyarihin ang nakaraang pag-iisip sapagkat ito ay nakibahagi sa talagang nangyayari. Ang talagang nangyayari ang pinakabuod at abottanaw ng pangyayari ng kasaysayan kung kaya't ang paglipas ng mga taong kumilala at kumilatis dito ay hindi katumbas ng pagwawakas ng pangyayari nito. Ang meron ay palaging meron, at ang tunay na palaisip ay laging nakakabit sa meron sa pangyayari nito sa kasysayan kung kaya't nagiging makasayasayan ang kanyang mga binigkas na nananawagan lagi ng muli't muling pag-uulit.

Ang pagbigkas sa meron ay pagiging makatotohanan, ang pagpaparaya sa pangyayari ng talagang nangyayari, ang pakikipagsapalaran sa saysay ng kasaysayang patuloy na humihiling ng masigasig na pagpupulis at pagdanas. Hindi sapat ang kumilos lamang sa mundo ng konsepto. Kailangang danasin at bigkasin ang talagang nangyayari sapagkat, "Ang pagbigkas sa meron ay pasiyang mabuhay sa talagang totoo: pasiyang kailangang alagaan at pairalin at iligtas sa mga pag-urong ng kahinaan."19 Bilang paksa ng mga paksa, nananawagan ang meron na patalabin ang bisa ng talagang totoo sa kasaysayan, at mangyayari lamang ito sa pagtalima, pakikinig, pagtataya, at pagbigkas sa meron mismo. Iisa ang sinasabi ng logos: na meron, at ang namimilosopiya ay tinatawag na ulitin ito nang ulitin sa parating bagong paraan sa bawat yugto ng kasaysayan.

\section{Kasaysayan sa Panahong Moderno: Pag-aalala sa Paglimot}

Ayon kay Heidegger, "Withdrawal is an event. In fact, what withdraws may even concern and claim man more essentially than anything present that strikes

${ }^{18}$ Roque J. Ferriols, "Fr. W. Norris Clarke, SJ: Heswitang Metapisiko," in Pagdiriwang sa Meron: A Festival of Thought Celebrating Roque J. Ferriols, SJ, ed. by Nemesio S. Que, SJ, at Agustin Martin Rodriguez (Quezon City: Office of Research and Publications, Ateneo de Manila University, 1997), 268.

${ }^{19}$ Ferriols, Pambungad sa Metapisika, 119.

(c) 2018 Marc Oliver D. Pasco

https://www.kritike.org/journal/issue 23/pasco december2018.pdf

ISSN 1908-7330

(c) $)$ BY-NC-ND 
and touches him." 20 Mula sa pagtanaw na ito, mainam pagmunihan kung anong ispesipikong kaganapan sa kasaysayan ang masasabing kanlungan ng nasabing paglimot. Sa palagay ni Heidegger, sa modernong panahon umabot sa rurok ng paglimot ang tao sa kasaysayan ng Sein. Sa Kartesyanong mundo ng mga bagay na may haba't sukat, pawang ang pagka-nabibilang at pagkanasusukat lamang ng mga nagmemeron ang siyang tinatanglawan ng kasaysayan. Kalkulasyon ang pangunahing patakaran ng pag-iisip at kumikilos ang tao sa daigdig na hubog ng kanyang mismong imahe. Mula sa Arkimedyanong punto na natukoy ni Descartes sa hindi maitatatwang pagiisip ng suheto, sumulong ang kasaysayan tungo sa progresibong pagkakawangis ng lahat ng nagpapakita ayon sa mga plano ng tao para sa kanyang sarili, na siyang naging pundasyon ng modernong teknolohiya. Ang pagkanauuna at pagkapinakatiyak na importante palagi ng cogito bilang fundamentum absolutum inconcussum veritatis ang nagdidikta ng abot-tanaw ng katotohanan ukol sa mga umiiral na nagpapakita. ${ }^{21}$ Ang suheto ang nagtatakada ng mga rasyunal na hangganan kung saan maaari lamang magpakita ang Sein. Ginagawa niya ito sa pamamagitan ng walang humpay na pagbibilang at pagsusukat na naglalayong iuwi sa pagkamasusukat at pagkamabibilang ang lahat sapagkat sa ganitong porma lamang ito kapakipakinabang para sa tao. Ngunit ayon kay Ferriols:

Sa lahat ng pagbilang at pagsukat, may paglayo sa meron na ginaganap. Hindi lamang sa masasalimuot na metodo ng matematika, kundi pati sa pinakasimpleng pagbilang at pagsukat, pati kung nakikita at nahahawakan ng isa isa ang mga binibilang at sinusukat, may paghiwalay sa meron na hindi maiiwasan; sapagkat kailangang tuonan lamang ng pansin ang pagka-maaaring-bilangin at pagka-maaaringsukatin ng mga bagay, at nalilimutan ng taga-sukat ang buong lalim at yaman $n g$ meron. ${ }^{22}$

Sa ganitong konteksto, masasabing nakalilimot ang tao sa misteryong pinagbubukalan ng lahat ng mga umiiral at nakatutok lamang ang kanyang atensyon sa mga aspeto ng umiiral na umaayon sa pagtatakda ng panahong moderno. Kumbaga, hindi sa ayaw ng taong danasin ang mismong meron, sadyang nanakubli lamang ang posibilidad na ito sa kanya dahil siya ay

${ }^{20}$ Martin Heidegger, What is Called Thinking, trans. by J. Glenn Gray (New York: Harper and Row Publishers, 1968), 9.

${ }^{21}$ Martin Heidegger, "The Age of the World Picture," in The Question Concerning Technology and Other Essays, trans. by William Lovitt (New York: Harper and Row Publishers, 1977), 148.

${ }^{22}$ Ferriols, Pambungad sa Metapisika, 170. Kay Ferriols ang italisasyon.

(C) 2018 Marc Oliver D. Pasco

https://www.kritike.org/journal/issue 23/pasco december2018.pdf

ISSN 1908-7330 
nabubuhay sa panahong modernong kung saan paglimot sa meron ang praktikal at usong paraan ng pag-iisip. Masasabing sa yugtong ito ng kasaysayan, ang cogito ang batayang-unibersal ng lahat ng uri ng pagpapakita ng mga umiiral.

Halimbawa, sa panahon ngayon, kapag bumiyahe ang tao patungo sa isang lugar na masasabing hindi pa inaabot ng "pag-unlad," hindi niya maiwasang makita ang mga bakanteng lupa mula sa lente ng paghahati-hati ng mga subdibisyon, o sa upa kada metro kuwadrado ng mga lupang nakatiwangwang na puwedeng tayuan ng mga gusaling pang-komersyo. $\mathrm{O}$ kaya naman, sa mundo ng akademya, bago magsulat ang isang mananaliksik, malay man siya o hindi, ay iniaayon niya ang paksa at metodolohiya ng kanyang sasaliksikin batay sa mga pamantayan ng posibilidad ng pondo para dito, at kung sa palagay niya'y wala naman siyang makukuhang isponsor para sa kanyang paksa ay papalitan niya ito o 'di kaya'y iaangkop ito sa mga kahilingan ng "kapondo-pondong" pananaliksik.

Sa madaling salita, sa ating modernong panahon, isinusulong ng iba't ibang sangay ng kaalamang maka-agham at teknolohikal ang isang uring pagtanaw na nagkakahon sa mga nagmemeron sa kuwadro ng pagkamaaaring-masukat at pagka-maaaring-mabilang sa layong isalin sa wika ng kalkulasyon ang logos ng meron. Pagkatiyak sa sariling mga ideya ang layunin ng ganitong pag-iisip at hindi ang pakikinig sa meron. Ang subiectum, ang yaong nauuna't nakalatag na bago pa ang lahat, ang basehan ng pagiging umiiral ng mga umiiral. Ito ang panahon ng paglimot sa meronang walang-malay na pananalamin ng suheto sa kanyang sarili sa lahat ng kanyang nakikita kung saan may mabalintunang alyenasyon ang nagaganap sa relasyon niya sa meron mismo. Dagdag pa ni Heidegger, "What is, in its entirety, is now taken in such a way that it first is in being and only is in being to the extent that it is set up by man, who represents and sets forth." ${ }^{23}$ Ang mismong pagmemeron, ang pagka-talaga ng nangyayari ay itinatalaga ng iba't ibang kilos-representasyon ng cogito sa sarili nito. Ang pangyayari ng nangyayari at ang kasaysayan ng pangyayari ng mga ito na naunawaan ng mga sinaunang Griyego bilang galaw mismo ng meron (bilang phusis, techné at alêtheia) na humihiling ng pagsabay at pakikibagay ay nalilimot at nauuwi sa mga kalkuladong numero at datos na handang magamit ng tao sa anumang paraan at panahon (Bestand). Ang nakakubling esensya ng Sein bilang pagprepresensya ng mga nagprepresensya ay nalilimot bilang siyang lihim na abot-tanaw ng saysay ng kasaysayan.

Sa bahagi ng kanyang libro kung saan pinag-usapan niya ang sozein ta phainomena ng penomenolohiya, nililinaw ni Ferriols na ang pagliligtas sa nagpapamasid ay hindi lamang ukol sa mga bagay na nagpapakita sa

${ }^{23}$ Heidegger, "The Age of the World Picture," 129-130.

(c) 2018 Marc Oliver D. Pasco

https://www.kritike.org/journal/issue 23/pasco december2018.pdf

ISSN 1908-7330

(c) $)$ BY-NC-ND 
kasalukuyan, kundi nangangailangan din ng pagtalima sa mga hindi pa nagpapakita o hindi pa napagmamasdan. Tunay na makasaysayan ang pagunawa ni Ferriols sa phainomena-nagpapasilip ito hindi lamang bilang nandiyan na o nandiyan na dati, kundi bilang hindi pa nagpapamasid, yaong hindi pa hinog sa panahon para mapansin at maisalba. Wika niya, "Bahagi rin ng sozein ta phainomena na hanapin at antabayanan at magpaka-alisto sa mga hindi pa napagmamasdan. Nasa sozein ta phainomena din ang pagkabukas ng loob sa data na darating pa." ${ }^{24}$ Kumikilos sa isang makasaysayang abot-tanaw ang puntong ito ni Ferriols. Bahagi ng pagpapakita ng nagpapakita ang pagiging dinamiko nito. Kung layon ng kalkulatibong pagiisip ng modernong panahon ang pagpapako sa ngayon ng kasaysayan, sa ganang ang ngayon ang laging handang mabilang, makalkula, at mapanghawakan, iminumungkahi ni Ferriols at ni Heidegger ang walang sawang pag-aantabay sa wala pa at sa hindi pa nagpapakita. Kailangang isalba ang mga posibilidad ng meron na natatabunan ng pagka-obheto nito sa harap ng suheto. Nananawagan ang pag-iisip na mamulat ito sa sarili nitong mga hangganan sa abot-tanaw ng meron. Kaya't nasabi ni Heidegger na, "Most thought-provoking in our time is that we are still not thinking.... that we are still not thinking stems from the fact that the thing itself that must be thought about turns away from man, has turned away long ago." ${ }^{25}$ Sinasabi ni Heidegger na tayo ay nabubuhay sa isang panahon kung saan napakahirap umusbong ang tunay na pag-iisip, hindi dahil hindi tayo nag-iisip, kung hindi dahil sadyang nagtatago ang dapat na pinag-uukulan ng ating pag-iisip at pagtalima.

Ano pa nga ba itong nagtatagong sadyang lampas sa abot-tanaw ng kasalukuyang uri ng pag-iisip kung hindi ang misteryosong pagtatakda ng Sein ng mismong paglimot na nangyayari sa yugtong ito ng kasaysayan. Sinususugan ng pag-iisip ni Heidegger ang punto ni Ferriols ukol sa pagbubukas-loob sa hindi pa nagpapakita. Wika niya, "The more original the thinking, the richer will be what is unthought in it. The unthought is the greatest gift that thinking can bestow." 26 Habang mas naiuugat ng nag-iisip ang sarili niya sa hindi-maiisip-at-hindi-pa-mabibigkas-na-nabigkas-na-rin-dati, mas nagiging mulat siya sa kanyang pagiging kabahagi ng kasaysayan ng talagang nangyayari. At ano ba ang talagang nangyayari ngayon, at mula pa noon-ang meron, at parating meron, maging sa panahon ng animo'y pagwawala ng tao sa kasaysayan. Kailangang ulitin ang pag-aalala sa paglimot ng kalimot-limot na talagang nangyayari sa kasaysayan sa pamamagitan ng tunay na pag-iisip, o sa wika ni Ferriols, sa pagbigkas sa meron. Sapagkat, wika nga ni Heidegger, "In order to bring into view what

${ }^{24}$ Ferriols, Pambungad sa Metapisika, 187.

${ }^{25}$ Heidegger, What is Called Thinking, 6-7.

${ }^{26}$ Ibid., 76.

(C) 2018 Marc Oliver D. Pasco https://www.kritike.org/journal/issue 23/pasco december2018.pdf ISSN 1908-7330 


\section{FERRIOLS AT HEIDEGGER}

resides in a visual field, the visual field must precisely light up first, so that it might illuminate what resides within it; however, it cannot and may not be seen explicitly. The field of view, alêtheia, must in a certain sense be overlooked." 27 Sa ganitong pagtanaw, ang kasaysayan ng paglimot ay hindi lamang nakaugat sa modernong kalkulatibong pag-iisip kung hindi sa mismong esensya ng katotohanang patuloy na nagkukubli sa kasaysayan. Dagdag pa ni Heidegger, "To recollectively think-upon what has-been is to fore-think into the unthought that is to be thought. To think is to recollectively fore-think. It neither dwells on what has-been as a past represented by historiography, nor is it a representational thinking that stares with prophetical pretenses into a supposedly known future. Thinking as a recollective fore-thinking is the leaping of the leap. This leap [Sprung] is a movement [Satz] to which thinking submits." 28 Nangangailangan ng isang paglundag patungo sa pag-aalala sa hindi pa nasasabi ang pakikipagsapalaran ng tao sa kasaysayan ng talagang nangyayari. Gaya ng sinabi ni Ferriols, kailangang isalba ang hindi pa rin nabibigkas, bilang sarili nito-bilang siyang hindi pa rin nabibigkas na dapat bigkasin. Ang paglundag na ito ay isang radikal na pag-aalala sa isang katotohanang hindi pa lantad sa tao sa modernong panahon, at kung gayo'y humihiling ng matinding pasensya at atensyon mula sa mga taong umaasang mabigkas ang totoo.

\section{Pangwakas}

Ang kasaysayan ng meron ay nangyayari lamang sa pag-aalala sa paglimot. Iiisa ang paksa ng lahat ng pagbigkas-ang meron, subalit kailangang maging mulat sa katotohanang sa bawat pagtatangkang bumaling sa totoo at maging makabuluhang bahagi ng kasaysayan, nandoon lagi ang hindi maiiwasang posibilidad ng paglimot dahil na rin sa mismong esensya ng meron. Bago pa man subuking bigkasin ang ipinahihiwatig ng meron sa kasaysayan, kailangan munang magpadala ang tao sa tawag ng meron na magpapamulat sa kanya na madalas kaysa hindi, walang masasabi sa harap ng kayamanan ng meron. Kailangang hayaang maging daluyan ng wika ng meron ang wika ng tao kung nais niyang bumigkas ng totoo. Sa wika ni Ferriols, "May pagbigkas ng salita na kusang pag-apaw ng biglang liwanag; nagiging sabay bunga at sanhi ng pag-uunawa ang ganiyang salita. May pagbigkas ng salita na pagtupad ng tao sa kanyang papel sa daigdig bilang taga-unawa at pag-uunawa. Ang ganyang pagbigkas ay hindi lamang nanggagaling sa tao bilang sangkap ng sansinukob, kundi isang kilos din ng

\footnotetext{
${ }^{27}$ Heidegger, Basic Questions of Philosophy: Selected "Problems" of "Logic," trans. by Richard Rojcewicz and André Schuwer (Indianapolis: Indiana University Press, 1994), 127-128.

${ }^{28}$ Heidegger, The Principle of Reason, 94.

(c) 2018 Marc Oliver D. Pasco

https://www.kritike.org/journal/issue 23/pasco december2018.pdf

ISSN 1908-7330
}

(cc) BY-NC-ND 
sansinukob na tumatalab sa loob ng tao." ${ }^{29}$ Hindi lamang isang instrumento ang salita na ginagamit ng tao para ipahayag ang kanyang saloobin. Hindi rin ito simpleng konsepto na kumakahon at nagbibigay-hugis at mukha sa mga umiiral para sa tao. "Language is the lighting-concealing arrival of being itself." 30 Sa wika nagaganap ang mismong pangyayari ng meron. Sa wika nasasangkot ang tao sa kasaysayan ng talagang nangyayari. Sa wika bumabakas ang kasaysayan. Kaya't kailangang ulit-ulitin ang pagbigkas, sapagkat hanggang meron, hanggang may nangyayari, patuloy ang pagkukubli ng meron sa kanyang kapayakan. Ang kasaysayan ng talagang nangyayari ay kailangang patuloy na danasin ng taong nakatadhanang makalimot sa kanyang lugar sa kasaysayan. Tunay na makasaysayan ang mga ginanap na pamimilosopiya nina Ferriols at Heidegger. Pareho nilang sinubukang buksan ang sarili sa paanyaya ng pakikipagsapalaran sa pangyayari ng talagang nangyayari. Hindi maluluma ang kanilang pamimillosopiya sapagkat ito ay nangyayari pa rin at mangyayari pa, hangga't may mga katulad nating naghahangad na masabi ang hindi masasabi.

Department of Philosophy, Ateneo de Manila University, Philippines

\section{References}

Ferriols, Roque, Mga Sinaunang Griyego (Quezon City: Office of Research and Publications, Ateneo de Manila University, 1991). . Pambungad sa Metapisika (Quezon City: Office of Research and Publications, Ateneo de Manila University, 1997). . "Fr. W. Norris Clarke, SJ: Heswitang Metapisiko," in Pagdiriwang sa Meron: A Festival of Thought Celebrating Roque J. Ferriols, SJ, ed. by Nemesio S. Que at Agustin Martin Rodriguez (Quezon City: Office of Research and Publications, Ateneo de Manila University, 1997).

Heidegger, Martin, An Introduction to Metaphysics, trans. by Ralph Manheim (New York: Yale University Press, 1959). . What is Called Thinking, trans. by J. Glenn Gray (New York: Harper and Row Publishers, 1968). . "A Dialogue on Language," in On the Way to Language, trans. by. Peter D. Hertz (New York: Harper and Row Publishers, 1971). . "The Anaximander Fragment," in Early Greek Thinking, trans. by David Farrell Krell at Frank A. Capuzzi (San Francisco: Harper San Francisco, 1975).

${ }^{29}$ Ferriols, Mga Sinaunang Griyego, 21.

${ }^{30}$ Heidegger, "Letter on Humanism," trans. by Frank A. Capuzzi, in Pathmarks, ed. by William McNeill (Cambridge. Cambridge University Rress, 1998), 249.

(c) 2018 Marc Oliver D. Pasco https://www.kritike.org/journal/issue 23/pasco december2018.pdf ISSN 1908-7330 


\section{FERRIOLS AT HEIDEGGER}

. "The Age of the World Picture," in The Question Concerning Technology and Other Essays, trans. by William Lovitt (New York: Harper and Row Publishers, 1977).

. "The Turning," in The Question Concerning Technology and Other Essays, trans. by William Lovitt (New York: Harper and Row Publishers, 1977).

. Basic Concepts, trans. by Gary E. Aylesworth (Indianapolis: Indiana University Press, 1993).

. Basic Questions of Philosophy: Selected "Problems" of "Logic," trans.

by Richard Rojcewicz and André Schuwer (Indianapolis: Indiana University Press, 1994).

. The Principle of Reason, trans. by Reginald Lilly (Indianapolis: Indiana University Press, 1996).

. "Letter on Humanism," trans. by Frank A. Capuzzi, om Pathmarks, ed. by William McNeill (Cambridge: Cambridge University Press, 1998).

Inwood, Michael, A Heidegger Dictionary (Malden: Blackwell Publishers, 1999).

Seidel, George, Joseph, Martin Heidegger and the Pre-Socratics (Nebraska: University of Nebraska Press, 1964). 